Discrete Comput Geom 33:231-247 (2005)

DOI: $10.1007 / \mathrm{s} 00454-004-1123-5$

\title{
Cutting Triangular Cycles of Lines in Space*
}

\author{
Boris Aronov, ${ }^{1}$ Vladlen Koltun, ${ }^{2}$ and Micha Sharir ${ }^{3}$ \\ ${ }^{1}$ Department of Computer and Information Science, Polytechnic University, \\ Brooklyn, NY 11201-3840, USA \\ aronov@cis.poly.edu \\ ${ }^{2}$ Computer Science Division, University of California, \\ Berkeley, CA 94720-1776, USA \\ vladlen@cs.berkeley.edu \\ ${ }^{3}$ School of Computer Science, Tel Aviv University, \\ Tel-Aviv 69978, Israel \\ michas@post.tau.ac.il \\ and \\ Courant Institute of Mathematical Sciences, New York University, \\ New York, NY 10012, USA
}

\begin{abstract}
We show that $n$ lines in 3-space can be cut into $O\left(n^{2-1 / 69} \log ^{16 / 69} n\right)$ pieces, such that all depth cycles defined by triples of lines are eliminated. This partially resolves a long-standing open problem in computational geometry, motivated by hidden-surface removal in computer graphics.
\end{abstract}

\section{Introduction}

The Problem. Let $\mathcal{L}$ be a collection of $n$ lines in $\mathbb{R}^{3}$ in general position. In particular, we assume that no two lines in $\mathcal{L}$ intersect and that the $x y$-projections of no two of the lines

\footnotetext{
* Part of the work on this paper has been carried out at the U.S.-Israeli Workshop on Geometric Algorithms, held in Jackson Hole, Wyoming, in the summer of 2002. Work on the paper by Boris Aronov and Micha Sharir has been supported by a joint grant from the U.S.-Israeli Binational Science Foundation. Work by Vladlen Koltun and Micha Sharir has also been supported by a grant from the Israel Science Fund (for a Center of Excellence in Geometric Computing). Work by Boris Aronov was also supported by NSF Grants CCR-9972568 and ITR CCR-00-81964. Work by Vladlen Koltun was also supported by NSF Grant CCR-01-21555. Work by Micha Sharir was also supported by NSF Grants CCR-97-32101 and CCR-00-98246, and by the Hermann Minkowski-MINERVA Center for Geometry at Tel Aviv University.
} 


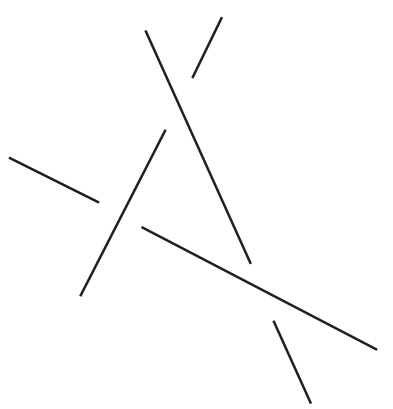

(a)

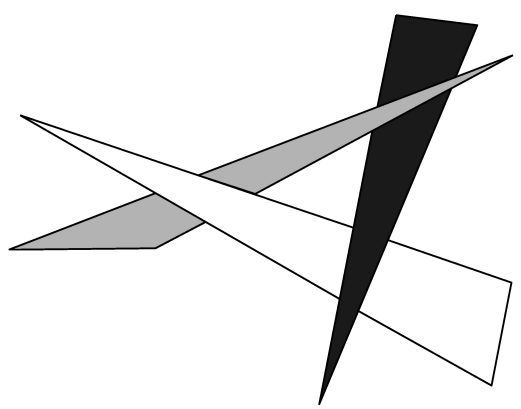

(b)

Fig. 1. Depth cycles formed (a) by three lines and (b) by three triangles

are parallel. For any pair $\ell, \ell^{\prime}$ of lines in $\mathcal{L}$, we say that $\ell$ passes above $\ell^{\prime}$ (equivalently, $\ell^{\prime}$ passes below $\ell$ ) if the unique vertical line $\lambda$ that meets both $\ell$ and $\ell^{\prime}$ intersects $\ell$ at a point that lies higher than its intersection with $\ell^{\prime}$. We denote this relation as $\ell^{\prime} \prec \ell$. The relation $\prec$ is total, but in general it need not be transitive and it can contain cycles of the form $\ell_{1} \prec \ell_{2} \prec \cdots \prec \ell_{k} \prec \ell_{1}$. We refer to $k$ as the length of the cycle. Cycles of length three are called triangular. See Fig. 1(a).

If we cut the lines of $\mathcal{L}$ at a finite number of points, we obtain a collection of lines, segments, and rays. We can extend the definition of the relation $\prec$ to the new collection in the obvious manner, except that it is now only a partial relation. Our goal is to cut the lines in such a way that $\prec$ becomes a partial order, in which case we call it a depth order. We note that it is trivial to construct a depth order with $\Theta\left(n^{2}\right)$ cuts: simply cut each line near every point whose $x y$-projection is a crossing point with another projected line. It is desirable to minimize the number of cuts. A long standing conjecture is that one can always construct a depth order with a subquadratic number of cuts. In this paper we make a step towards establishing this conjecture.

Background. The main motivation for studying this problem comes from hidden surface removal (HSR) in computer graphics. Given a collection of objects in $\mathbb{R}^{3}$, say pairwise disjoint triangles, and a viewing point, placed for convenience at $z=-\infty$, we wish to compute and render all visible portions of the input objects; that is, for each object $o$ we wish to compute the subset of all points $p$ on $o$ for which the downward-directed ray emanating from $p$ meets no other object.

Until the 1970s, HSR was considered one of computer graphics' most important problems, and has received a substantial amount of attention; see [21] for a survey of the ten leading HSR algorithms circa 1974. Since then it has been solved in hardware, using the $z$-buffer technique [4], which produces a "discrete" solution to the problem, by computing the nearest object at each pixel of the image. Nevertheless, there is still considerable interest in obtaining an object-space representation of the visible scene, which is a combinatorial description of the visible portions, independent of the pixel locations and resolution. 
These considerations motivated an extensive study of HSR in computational geometry, culminating in the early 1990s with a number of algorithms that provide both conceptual simplicity and satisfactory running-time bounds. See [2] and [9] for overviews of these developments, and [14] for a simple HSR algorithm with good theoretical running-time bounds.

A common feature of most HSR algorithms is that they rely on the existence of a consistent depth order for the input objects, which is defined as in the case of lines: a pair of objects $A, B$ satisfy $A \prec B$ if there exists a point on $B$ so that the downwarddirected ray emanating from it meets $A$. These algorithms begin by sorting the objects either front-to-back (e.g., the Overmars-Sharir algorithm [14]) or back-to-front (e.g., the classical Painter's algorithm [21]).

A large number of algorithms have been developed for testing whether the relation $\prec$ in a collection of triangles is an order; see [2] and the references therein. However, while these algorithms help detect cycles, they do not provide strategies for dealing with cycles, such as the one shown in Fig. 1(b).

One such common strategy is to eliminate all depth cycles, by cutting the objects into portions that do not form cycles, and running an HSR algorithm on the resulting collection of pieces. In 1980 Fuchs et al. [10] introduced binary space partition (BSP) trees, which can be used to perform the desired cutting. However, a BSP tree may force up to a quadratic number of cuts [16], which brings us back to the original challenge: devise an algorithm that, given a specific viewpoint and a collection of $n$ triangles in $\mathbb{R}^{3}$, removes all depth cycles defined by this collection with respect to the viewpoint, using a subquadratic number of cuts. This has been an open problem since 1980.

In this paper we study the simpler problem mentioned above, by restricting the input to lines in space, rather than triangles. Note that since any cycle defined by a collection of line segments is also a cycle in the collection of lines spanned by these segments, the case of line segments is simpler than the case of lines, and we thus concentrate on the latter case. (The case of triangles, though, is more involved, since a depth cycle among triangles does not necessarily imply a depth cycle among their edges.)

The work of Solan [19] and of Har-Peled and Sharir [12] supplies algorithms that achieve the above goal, provided a subquadratic number of cuts is always sufficient. In particular, these works present algorithms that, given a collection $\mathcal{L}$ of $n$ lines (or segments) in 3-space, perform close to $O(n \sqrt{C})$ cuts (the precise bound is $O\left(n^{1+\varepsilon} \sqrt{C}\right)$ for [19] and $O(n \sqrt{C} \alpha(n) \log n)$ for [12]) that eliminate all cycles defined by $\mathcal{L}$ as seen from $z=-\infty$, where $C$ is the minimal required number of such cuts. That is, if we can provide a subquadratic bound on the minimal number of cuts that suffice to eliminate all cycles defined by a collection of lines, then the aforementioned algorithms are guaranteed to find a collection of such cuts of (potentially larger but still) subquadratic size.

Such an upper bound has however remained elusive. The only progress in this direction is due to Chazelle et al. [5], who in 1992 have analyzed the following special case of the problem. A collection of line segments in the plane is said to form a grid if it can be partitioned into two subcollections of "red" and "blue" segments, such that all red (resp., blue) segments are pairwise disjoint, and all red (resp., blue) segments intersect all blue (resp., red) segments in the same order; see Fig. 2. Chazelle et al. [5] have shown that if the $x y$-projections of a collection of $n$ segments in 3-space form a grid, then all 


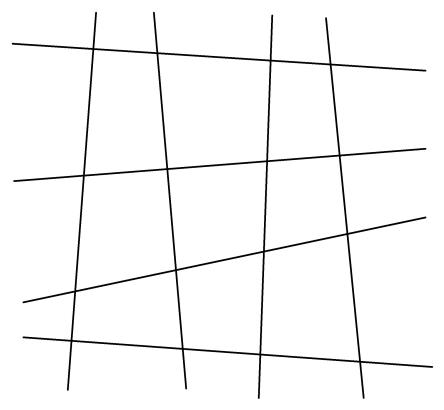

Fig. 2. A collection of line segments that forms a grid.

cycles defined by this collection (again, as seen from $z=-\infty$ ) can be eliminated with $O\left(n^{9 / 5}\right)$ cuts.

Our Contribution. This paper describes the first step towards obtaining subquadratic general upper bounds on the number of cuts that are sufficient to eliminate all cycles defined by an arbitrary collection of lines in space. Specifically, we show that all triangular cycles can be eliminated with $O\left(n^{2-1 / 69} \log ^{16 / 69} n\right)$ cuts. This allows adapting the technique of Har-Peled and Sharir [12], or of Solan [19], to yield an algorithm that eliminates all such cycles using close to $O\left(n^{2-1 / 138}\right)$ cuts.

While our main bound is still far from the lower bound $\Omega\left(n^{3 / 2}\right)$ provided by Chazelle et al. [5] and does not immediately apply to cycles of arbitrary length, it is an essential first step towards the complete solution. As the first nontrivial general upper bound for this problem, since the problem's conception more than 20 years ago, we expect it to be generalized and improved, and the techniques we introduce to be extended and simplified. A central component in our proof is a result of independent interest concerning the unrealizability of a certain "weaving pattern" of lines; see the next section for definitions.

\section{The Magen-David Weaving}

A weaving is a finite collection of lines drawn in the plane, such that at each intersection of a pair of lines, it is specified which of the two passes above the other. A weaving $\Psi$ is said to be realizable if there is a collection $\mathcal{L}$ of lines in 3-space (called the realization of $\Psi$ ) whose $x y$-projection forms a collection of lines that is combinatorially equivalent to the one that defines $\Psi$, and the lines in $\mathcal{L}$ adhere to the above-below constraints specified by $\Psi$. Otherwise, $\Psi$ is said to be unrealizable. A growing, albeit still relatively small, body of research deals with the analysis and classification of realizable and unrealizable weavings [11], [15], [17]. While it can be shown that, for a sufficiently large number of lines, most weavings are unrealizable, proving the unrealizability of a specific weaving is a rather nontrivial problem. We contribute to this study by describing a simple weaving of six lines and showing it not to be realizable. This result plays a crucial role in the overall analysis. 


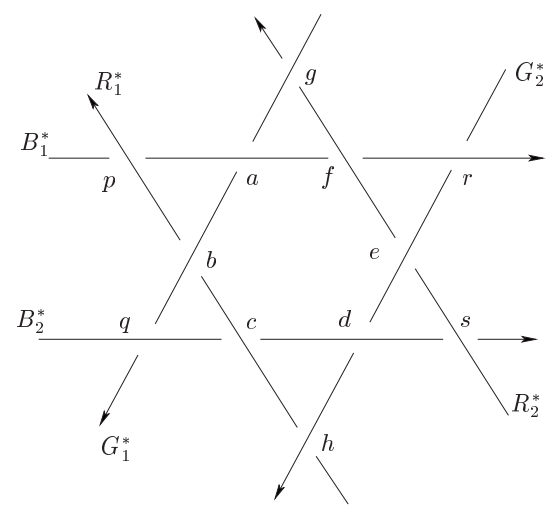

Fig. 3. The Magen-David weaving.

Consider the configuration shown in Fig. 3. It consists of two lines $B_{1}^{*}, B_{2}^{*}$, each crossing four other lines ${ }^{1} R_{1}^{*}, R_{2}^{*}, G_{1}^{*}, G_{2}^{*}$. All eight crossings occur on the boundary of a single wedge $W$ formed between $B_{1}^{*}$ and $B_{2}^{*}$. (The figure shows pairs of parallel lines, but this is drawn only for convenience. For example, it is immaterial whether $B_{1}^{*}$ and $B_{2}^{*}$ intersect to the left or to the right of the figure, and which of the lines $B_{1}, B_{2}$ passes above which in 3-space.) Moreover, $B_{1}^{*}$ meets the four lines in the order $R_{1}^{*}, G_{1}^{*}, R_{2}^{*}, G_{2}^{*}$, and $B_{2}^{*}$ meets them in the order $G_{1}^{*}, R_{1}^{*}, G_{2}^{*}, R_{2}^{*}$. Thus the only pairs of the four lines that cross within $W$ are $\left(R_{1}^{*}, G_{1}^{*}\right)$ and $\left(R_{2}^{*}, G_{2}^{*}\right)$. We also assume that the pair $\left(R_{1}^{*}, G_{2}^{*}\right)$ crosses on the opposite side of $B_{2}^{*}$, and that the pair $\left(R_{2}^{*}, G_{1}^{*}\right)$ crosses on the opposite side of $B_{1}^{*}$. Finally, we assume that the actual lines in 3-space, $B_{1}, B_{2}, R_{1}, R_{2}, G_{1}, G_{2}$, whose projections form the configuration, are such that the lines $G_{i}$ are above the lines $R_{j}$ at the appropriate four intersection points, the lines $R_{i}$ are above the lines $B_{j}$, and the lines $B_{i}$ are above the lines $G_{j}$, for $i, j=1,2$. Note that each of the eight triples $\left(B_{i}, R_{j}, G_{k}\right)$, for $i, j, k=1,2$, is a triangular cycle in the collection of these six lines. The weaving described is referred to as the Magen-David weaving. ${ }^{2}$ In light of the recent developments on the subject of weavings [11], [15], [17], the following result is of independent interest.

\section{Theorem 2.1. The Magen-David weaving is unrealizable.}

Proof. Assume to the contrary that there exist six lines $B_{1}, B_{2}, R_{1}, R_{2}, G_{1}, G_{2}$ in 3space that realize the Magen-David weaving, and whose $x y$-projections are, respectively, $B_{1}^{*}, B_{2}^{*}, R_{1}^{*}, R_{2}^{*}, G_{1}^{*}, G_{2}^{*}$. We assume that the figure is drawn in a coordinate frame in which $B_{1}^{*}$ lies above $B_{2}^{*}$ (in the $y$-direction) within $W$, as in Fig. 3 .

\footnotetext{
${ }^{1}$ We use the notation $\ell^{*}$ for the $x y$-projection of a line $\ell$ in 3 -space. In accordance with this, we denote lines drawn in the $x y$-plane using the $*$-notation. The above/below data at a crossing is depicted in the figures using the standard convention for views from above that the line passing below is drawn with a small gap around the crossing.

2 "Magen-David" is the original Hebrew expression for the Star of David, meaning literally "David's Shield."
} 


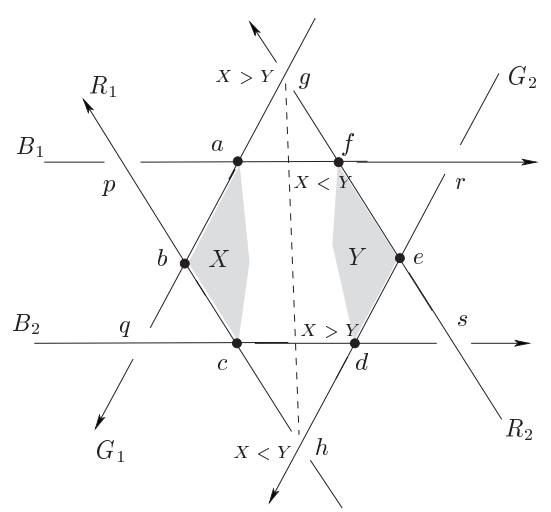

Fig. 4. An (impossible) realization of the Magen-David weaving, after six contacts were enforced.

We also use the following notation. When two projected lines meet at a point $w$, each of the original lines contains a point that projects to $w$. We designate these two points as $w_{\xi}, w_{\eta}$, where $\xi, \eta \in\{B, R, G\}$ denote the families of the respective lines. For example, $R_{1}^{*}$ and $G_{1}^{*}$ meet at $b$, and the corresponding point on $R_{1}$ (resp., $G_{1}$ ) is denoted by $b_{R}$ (resp., $b_{G}$ ). In the proof below we rotate some of the lines (without changing their $x y$-projections) to make certain pairs of lines touch. When this happens, we denote, with a slight abuse of notation, their common point by the symbol denoting its projection.

Rotate the line $G_{1}$, without changing its vertical projection, about $a_{G}$, such that the part of $G_{1}$ whose $x y$-projection is incident to the central hexagon in the weaving rotates downwards. Clearly, it will meet the line $R_{1}$ over the point $b$ before crossing any other line; we stop the rotation of $G_{1}$ when this contact with $R_{1}$ occurs. Now rotate $R_{1}$ about $b$, again rotating the part of $R_{1}$ whose projection overlaps the hexagon downwards. It will similarly meet $B_{2}$ over $c$. Continue this process in a domino-like fashion, rotating $B_{2}$ until it meets $G_{2}$, then $G_{2}$ until it meets $R_{2}$, then $R_{2}$ until it meets $B_{1}$, and finally $B_{1}$ until it meets $G_{1}$. We have thus forced six pairs of lines to touch each other, without changing their vertical projections. The resulting configuration is shown in Fig. 4, where dots denote contacts. Note that the above process creates no contacts between the lines other than the six contacts described above, and does not change any other above/below relationships between the lines.

Let $X$ denote the plane spanned by the contact points $a, b, c$ and let $Y$ denote the plane spanned by the contact points $d, e, f$ in $\mathbb{R}^{3}$. Note that $X$ also contains the points $g_{G}, h_{R}, p_{R}$ and $q_{G}$, and $Y$ similarly contains $g_{R}, h_{G}, r_{G}$, and $s_{R}$. Since $p_{B}$ lies below $p_{R}$, the line $B_{1}$ passes below $X$ over $p$. Since $B_{1}$ meets $X$ at $a$ we conclude that to the right of $a, B_{1}$ lies above $X$. Symmetrically, $r_{B}$ lies above $r_{G}$, so $B_{1}$ passes above $Y$ over $r$, meets $Y$ at $f$, and thus lies below $Y$ to the left of $f$. Hence, between $a$ and $f, B_{1}$ lies above $X$ and below $Y$, so $X$ lies below $Y$ over this interval.

A symmetric analysis applies to $B_{2}: q_{B}$ lies above $q_{G}$, so $B_{2}$ lies above $X$ over $q$, meets $X$ at $c$, and thus lies below $X$ to the right of $c$. On the other hand, $s_{B}$ lies below $s_{R}$, so $B_{2}$ lies below $Y$ over $s$, meets $Y$ at $d$, and thus lies above $Y$ to the left of $d$. Hence, $X$ lies above $Y$ over the interval between $c$ and $d$. 
Since $g_{G}$ lies above $g_{R}, X$ lies above $Y$ over $g$. Since $h_{R}$ lies below $h_{G}, X$ lies below $Y$ over $h$. Consider $X$ and $Y$ as linear functions defined over the interval $g h$. Our assumptions imply that $B_{1}^{*}$ (resp., $B_{2}^{*}$ ) crosses $g h$ at a point that lies in the interval $a f$ (resp., $c d$ ). Hence, $X$ lies above $Y$ over $g$, below $Y$ over $B_{1}^{*} \cap g h$, above $Y$ over $B_{2}^{*} \cap g h$, and below $Y$ over $h$. This alternation is impossible for a pair of linear functions, implying that the Magen-David weaving is unrealizable.

Remarks. (1) Notice that the contradiction is reached even by using only one of the two above/below relationships of the lines over $g$ and over $h$. We thus obtain a slightly stronger unrealizability result, in which one of these order relationships can be arbitrary.

(2) Theorem 2.1 is a central tool in our analysis of the number of cuts needed to eliminate all triangular cycles. It is interesting to note that the previous result of Chazelle et al. [5] also relies on the unrealizability of a weaving, which in that case was the complete $4 \times 4$ weaving [15].

\section{Eliminating All Triangular Cycles}

Let $\mathcal{L}$ be a set of $n$ nonvertical lines in 3-space in general position and let $\mathcal{L}^{*}=\left\{\ell^{*} \mid\right.$ $\ell \in \mathcal{L}\}$ denote the set of their projections. A cycle $c$ in $\mathcal{L}$ of the form $\ell_{1} \prec \ell_{2} \prec \cdots \prec$ $\ell_{j} \prec \ell_{1}$ can be represented as a closed oriented (possibly self-intersecting or even selfoverlapping) polygonal path $c^{*}=p_{1} p_{2} \cdots p_{n} p_{1}$, where $p_{i}$ is the intersection point of $\ell_{i}^{*}$ and $\ell_{i+1(\bmod j)}^{*}$.

A triangular cycle is defined by three lines $\ell_{1}, \ell_{2}, \ell_{3}$ satisfying $\ell_{1} \prec \ell_{2} \prec \ell_{3} \prec \ell_{1}$. We call a triangular cycle $c$ clockwise (resp., counterclockwise) if the resulting orientation of $c^{*}$ as we trace it in the order $\ell_{1}^{*} \rightarrow \ell_{2}^{*} \rightarrow \ell_{3}^{*} \rightarrow \ell_{1}^{*}$ is clockwise (resp., counterclockwise); see Fig. 5.

From Triangular Cycles to Empty Triangular Cycles. In this paper we confine our study to triangular cycles; thus from now on, the unqualified term "cycle" will always refer to a triangular cycle. We wish to cut the lines in $\mathcal{L}$ so that all such cycles are eliminated. Here is a simple procedure that achieves this goal. Fix a parameter $k$ to be determined later. For each $\ell \in \mathcal{L}$, cut $\ell$ at (the points projecting on) every $k$ th vertex of the arrangement $\mathcal{A}\left(\mathcal{L}^{*}\right)$ lying on $\ell^{*}$. The total number of cuts is $O\left(n^{2} / k\right)$. After these cuts are performed, any cycle $c$ that has not been eliminated has the property that $c^{*}$ is crossed by at most
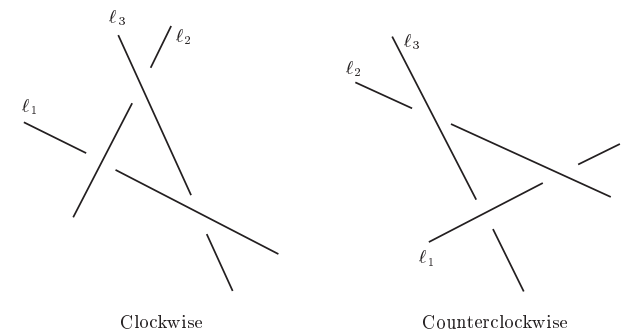

Fig. 5. The two types of triangular cycles. 
$3 k / 2$ lines of $\mathcal{L}^{*}$. Using the probabilistic analysis technique of Clarkson and Shor [8], the overall number of these "light" triangular cycles is $O\left(k^{3} v_{0}(n / k)\right)$, where $v_{0}(m)$ is the maximum number of triangular cycles $c$ in a collection of $m$ lines in space, such that $c^{*}$ is a face in the arrangement of the projected lines. (We refer to cycles of the latter type as empty.) Hence, the following number of cuts is certainly sufficient for eliminating all triangular cycles in $\mathcal{L}$ :

$$
O\left(\frac{n^{2}}{k}+k^{3} v_{0}\left(\frac{n}{k}\right)\right) .
$$

Let $C$ be a family of triples $\left(\ell_{1}, \ell_{2}, \ell_{3}\right)$ of distinct lines of $\mathcal{L}$, such that each triple in $C$ forms a counterclockwise triangular cycle whose $x y$-projection is a face of $\mathcal{A}\left(\mathcal{L}^{*}\right)$. We refer to such cycles as (counterclockwise) empty cycles. It suffices to obtain a bound on $|C|$, since, by symmetry, the overall number of triangular empty cycles is at most twice this bound. This is precisely what we do in Sections 4 and 5, which culminate in Theorem 5.1. The theorem states that $|C|$ is bounded by $O\left(n^{2-1 / 34} \log ^{8 / 17} n\right)$, which implies that $v_{0}(n)=O\left(n^{2-1 / 34} \log ^{8 / 17} n\right)$. Plugging this estimate into (1) we conclude that the number of cuts needed to eliminate all triangular cycles in $\mathcal{L}$ is

$$
O\left(\frac{n^{2}}{k}+k^{3}\left(\frac{n}{k}\right)^{2-1 / 34} \log ^{8 / 17} \frac{n}{k}\right)=O\left(\frac{n^{2}}{k}+k^{35 / 34} n^{2-1 / 34} \log ^{8 / 17} n\right) .
$$

Choosing $k=n^{1 / 69} / \log ^{16 / 69} n$, we obtain the main result of this paper.

Theorem 3.1. A set $\mathcal{L}$ of $n$ nonvertical lines in $\mathbb{R}^{3}$ in general position can be cut into $O\left(n^{2-1 / 69} \log ^{16 / 69} n\right)$ segments and rays, such that no triangular cycles are present in the depth order of these portions of the lines.

The remainder of the paper is devoted to the derivation of the bound $O\left(n^{2-1 / 34} \log ^{8 / 17} n\right)$ on the number of empty counterclockwise triangular cycles.

\section{Empty Cycles in a Restricted Setting}

We commence our analysis of empty cycles by first proving a subquadratic bound on their number in a restricted setting. In the next section we describe a reduction of the analysis of empty cycles in a general collection of lines to the case considered here.

Lemma 4.1. Let $\mathcal{L}$ be the disjoint union of three collections of lines: $B$ (blue lines), $R$ (red lines), and $G$ (green lines), that satisfy the following two conditions:

(C1) Each line in $B$ passes below each line in $R$, which passes below each line in $G$, which passes below each line in B. In particular, for any $b \in B, r \in R$, and $g \in G$, the triple $b, r, g$ forms a cycle.

(C2) The lines in $\mathcal{L}^{*}$ are oriented and there exist three orientations $\alpha, \beta, \gamma$ such that (1) $\alpha, \beta, \gamma,-\alpha,-\beta,-\gamma$ (where $-\theta$ denotes the orientation antipodal to orientation $\theta$ ) are distinct and occur in this counterclockwise cyclic order on the circle of orientations, and (2) the directions of the lines of $B^{*}, R^{*}, G^{*}$ occur in the open intervals $(\alpha, \beta),(\gamma,-\alpha),(-\beta,-\gamma)$, respectively. See Fig. 6. 


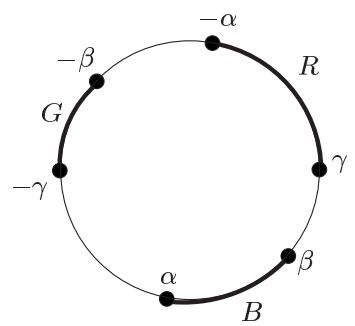

Fig. 6. Illustration of condition (C2); $B, R, G$ are used to label the intervals containing the directions of the corresponding projected lines.

Let $C$ be a collection of cycles defined by $\mathcal{L}$ such that each cycle of $C$ projects to a triangular face of $\mathcal{A}\left(\mathcal{L}^{*}\right)$ that is bounded by a line of $B^{*}$, a line of $R^{*}$, and a line of $G^{*}$, and lies to the left of each of these oriented lines. Then the number of cycles in $C$ is

$$
O\left(|R|^{5 / 6}|B|^{1 / 3}|G|^{1 / 3} \min \{|B|,|G|\}^{1 / 3}+|R|^{1 / 2} \max \{|B|,|G|\}+|R|\right) .
$$

Observe that if $|\mathcal{L}|=n$ then this bound is $O\left(n^{11 / 6}\right)$, which is considerably stronger than the bound $O\left(n^{2-1 / 34} \log ^{8 / 17} n\right)$ mentioned above and established in Theorem 5.1 below for the general case. Note also that the lemma yields two additional similar bounds, obtained by cyclically permuting $B, R$, and $G$.

Proof. We assume that $|B| \leq|G|$, which involves no loss of generality, and establish the bound

$$
O\left(|R|^{5 / 6}|B|^{2 / 3}|G|^{1 / 3}+|R|^{1 / 2}|G|+|R|\right) .
$$

For the ensuing discussion, we assume, without loss of generality, that all lines of $R^{*}$ form angles of at most $\pi / 4$ with the $x$-axis, and are oriented from left to right; this can be enforced by an appropriate rotation and scaling of the coordinate frame. Put $b=|B|$, $r=|R|, g=|G|$. Fix a threshold parameter $t$, to be determined later. Let $R^{+}$denote the set of red lines that participate in at least $t$ cycles of $C$. The total number of cycles of $C$ that involve red lines in $R \backslash R^{+}$is at most $r t$. For each pair of lines $\rho_{1}, \rho_{2} \in R^{+}$, define the distance $d\left(\rho_{1}, \rho_{2}\right)$ to be the number of blue-green vertices of the arrangement $\mathcal{A}\left(\mathcal{L}^{*}\right)$ that lie in the double wedge $W\left(\rho_{1}, \rho_{2}\right)$ formed between the projections $\rho_{1}^{*}, \rho_{2}^{*}$ of these two lines and not containing the vertical ( $y$-parallel) direction. Assign to each line $\rho \in R^{+}$the sequence $C(\rho)$ of the cycles of $C$ whose $x y$-projections contain portions of $\rho^{*}$. These portions of $\rho^{*}$ form a sequence of pairwise disjoint segments of $\rho^{*}$, sorted from left to right along $\rho^{*}$.

Fix a pair $\rho_{1}, \rho_{2}$ of distinct lines in $R^{+}$. The intersection point $q$ of their projections splits each of the sequences $C\left(\rho_{1}\right), C\left(\rho_{2}\right)$ into two respective subsequences $C_{\mathrm{L}}\left(\rho_{1}\right), C_{\mathrm{R}}\left(\rho_{1}\right)$, and $C_{\mathrm{L}}\left(\rho_{2}\right), C_{\mathrm{R}}\left(\rho_{2}\right)$, where $C_{\mathrm{L}}\left(\rho_{i}\right)$ (resp., $\left.C_{\mathrm{R}}\left(\rho_{i}\right)\right)$ is the subsequence consisting of the cycles that precede (resp., succeed) $q$ along $\rho_{i}$, for $i=1,2$; note that no cycle on either line can contain $q$. Put $t_{1 \mathrm{~L}}=\left|C_{\mathrm{L}}\left(\rho_{1}\right)\right|, t_{1 \mathrm{R}}=\left|C_{\mathrm{R}}\left(\rho_{1}\right)\right|, t_{2 \mathrm{~L}}=\left|C_{\mathrm{L}}\left(\rho_{2}\right)\right|$, 


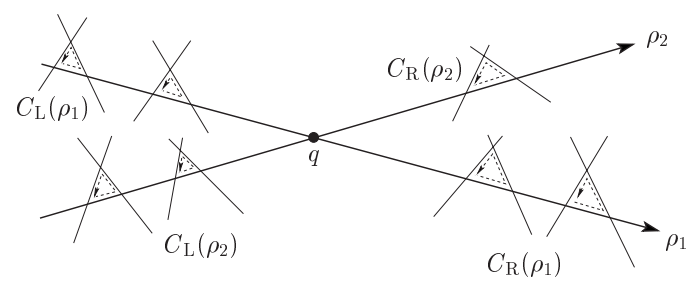

Fig. 7. The structure of the double wedge $W\left(\rho_{1}, \rho_{2}\right)$ and the cycles along it.

and $t_{2 \mathrm{R}}=\left|C_{\mathrm{R}}\left(\rho_{2}\right)\right|$. Suppose finally, without loss of generality, that $\rho_{2}$ lies counterclockwise of $\rho_{1}{ }^{3}$ See Fig. 7.

Claim 1. $W\left(\rho_{1}, \rho_{2}\right)$ contains at least $\frac{1}{2} t_{1 \mathrm{R}}^{2}+\frac{1}{2} t_{2 \mathrm{~L}}^{2}$ blue-green vertices; that is,

$$
d\left(\rho_{1}, \rho_{2}\right) \geq \frac{1}{2}\left(t_{1 \mathrm{R}}^{2}+t_{2 \mathrm{~L}}^{2}\right) .
$$

Proof. We show that the right wedge $W_{\mathrm{R}}\left(\rho_{1}, \rho_{2}\right)$ between $\rho_{1}^{*}$ and $\rho_{2}^{*}$ contains at least $\frac{1}{2} t_{1 \mathrm{R}}^{2}$ blue-green vertices. A symmetric argument implies that the left wedge $W_{\mathrm{L}}\left(\rho_{1}, \rho_{2}\right)$ contains at least $\frac{1}{2} t_{2 \mathrm{~L}}^{2}$ such vertices.

Let $c_{1}, c_{2}$ be two cycles in $C_{\mathrm{R}}\left(\rho_{1}\right)$, so that $c_{1}^{*}$ precedes $c_{2}^{*}$ along $\rho_{1}^{*}$; see Fig. 8. By the separation of orientations (condition (C2)), the blue line $\beta_{1}$ of $c_{1}$ and the green line $\gamma_{2}$ of $c_{2}$ must be such that $\beta_{1}^{*}$ and $\gamma_{2}^{*}$ cross to the left of $\rho_{1}^{*}$, at some blue-green vertex $v$. Note that $\rho_{2}^{*}$ does not intersect $c_{1}^{*}$ or $c_{2}^{*}$, because they are faces of $\mathcal{A}\left(B^{*} \cup R^{*} \cup G^{*}\right)$. If $v$ lay outside $W_{\mathrm{R}}\left(\rho_{1}, \rho_{2}\right)$, as in the figure, then $\rho_{2}^{*}$ would have had to cross the lines $\gamma_{1}^{*}, \beta_{1}^{*}, \gamma_{2}^{*}, \beta_{2}^{*}$ in this order. This, together with condition (C2), would have implied that the lines $\rho_{1}^{*}, \rho_{2}^{*}, \beta_{1}^{*}, \gamma_{1}^{*}, \beta_{2}^{*}, \gamma_{2}^{*}$ form an impossible weaving Magen-David configuration.

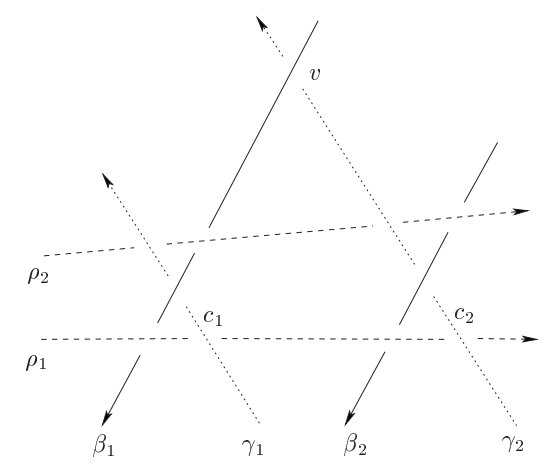

Fig. 8. The blue-green vertex $v$ must lie in $W\left(\rho_{1}, \rho_{2}\right)$.

\footnotetext{
${ }^{3}$ We say that an oriented line $\lambda$ in the plane lies counterclockwise (resp., clockwise) of another oriented line $\lambda^{\prime}$ if the counterclockwise angle from the direction of $\lambda$ to that of $\lambda^{\prime}$ is greater than $\pi$ (resp., less than $\pi$ ).
} 


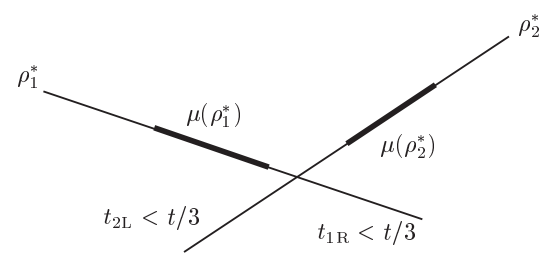

Fig. 9. $\mu\left(\rho_{1}^{*}\right)$ and $\mu\left(\rho_{2}^{*}\right)$ lie on the upper envelope of $\rho_{1}^{*}, \rho_{2}^{*}$.

Hence, every vertex $v$ of this type lies inside $W_{\mathrm{R}}\left(\rho_{1}, \rho_{2}\right)$. Adding the $t_{1 \mathrm{R}}$ blue-green vertices of the cycles themselves, which also lie in $W_{R}\left(\rho_{1}, \rho_{2}\right)$, we obtain a total of $\left(\begin{array}{c}t_{\mathrm{R}} \\ 2\end{array}\right)+t_{1 \mathrm{R}} \geq \frac{1}{2} t_{1 \mathrm{R}}^{2}$ blue-green vertices. This completes the proof of the claim.

Fix a line $\rho_{0} \in R^{+}$and consider the cluster $N\left(\rho_{0}\right)$ of lines $\rho \in R^{+}$such that $d\left(\rho, \rho_{0}\right)<t^{2} / 36$. The distance $d(\cdot, \cdot)$ satisfies the triangle inequality; this can be verified either directly or by using duality (see below for more details). Let $\rho_{1}, \rho_{2} \in N\left(\rho_{0}\right)$. We thus have

$$
d\left(\rho_{1}, \rho_{2}\right) \leq d\left(\rho_{1}, \rho_{0}\right)+d\left(\rho_{2}, \rho_{0}\right)<\frac{t^{2}}{18} .
$$

The above claim implies that (assuming that $\rho_{2}$ lies counterclockwise of $\rho_{1}$ )

$$
\frac{1}{2} t_{1 \mathrm{R}}^{2}+\frac{1}{2} t_{2 \mathrm{~L}}^{2}<\frac{t^{2}}{18}
$$

therefore $t_{1 \mathrm{R}}$ and $t_{2 \mathrm{~L}}$ are both smaller than $t / 3$. In other words, $\rho_{1}^{*}$ passes below (in the $y$-direction) the middle portion $\mu\left(\rho_{2}^{*}\right)$ of $\rho_{2}^{*}$, which is the shortest interval along $\rho_{2}^{*}$ that contains the portions of $\rho_{2}^{*}$ that participate in the middle $t / 3$ cycles along that line, and, symmetrically, $\rho_{2}^{*}$ passes below the middle portion $\mu\left(\rho_{1}^{*}\right)$ of $\rho_{1}^{*}$. This implies that $\mu\left(\rho_{1}^{*}\right)$ and $\mu\left(\rho_{2}^{*}\right)$ lie on the upper envelope of $\rho_{1}^{*}$ and $\rho_{2}^{*}$ (relative to the $y$-direction); see Fig. 9.

Applying this argument to each pair of lines in $N\left(\rho_{0}\right)$, we conclude that all the middle portions $\mu\left(\rho^{*}\right)$, for $\rho \in N\left(\rho_{0}\right)$, lie on the upper envelope $E$ of the projections of the lines in $N\left(\rho_{0}\right)$. Hence, $E$ contains the red portions of at least $t / 3\left|N\left(\rho_{0}\right)\right|$ cycles of $C$. However, since $E$ is a convex chain, a blue or green line can generate at most one cycle along $E$, as it intersects $E$ at just one point, due to the separation of orientations in condition (C2). Hence

$$
\frac{t}{3}\left|N\left(\rho_{0}\right)\right| \leq \min \{b, g\}=b \quad \text { or } \quad\left|N\left(\rho_{0}\right)\right| \leq \frac{3 b}{t} .
$$

We now construct clusters of this type iteratively, picking a line $\rho \in R^{+}$not belonging to any previously constructed cluster, and forming its cluster $N(\rho)$, using only lines that have not yet been assigned to any cluster. Let $R_{\mathrm{c}}$ denote the set of "centers" of these clusters, i.e., the lines $\rho$ with respect to which the clusters $N(\rho)$ have been defined. By construction, any pair of lines $\rho_{1}, \rho_{2} \in R_{\mathrm{c}}$ satisfies $d\left(\rho_{1}, \rho_{2}\right) \geq t^{2} / 36$.

We apply a standard duality transform to the $x y$-plane which preserves the abovebelow relationship (see [3]). We denote the dual of an object $a$ by $\tilde{a}$ to avoid confusion 
with the notation $a^{*}$ used to denote $x y$-projections. We obtain a set $\widetilde{R_{\mathrm{c}}^{*}}$ of red points, dual to the projections of the red lines in $R_{\mathrm{c}}$. Each blue-green vertex $v$ is mapped to the line connecting the corresponding dual blue and green points. Let $K$ denote this set of dual lines. A vertex $v$ lies in the double wedge $W\left(\rho_{1}, \rho_{2}\right)$ if and only if the line $\tilde{v}$ separates $\widetilde{\rho}_{1}^{*}$ and $\tilde{\rho}_{2}^{*}$. That is, $\underset{\sim}{d}\left(\rho_{1}, \rho_{2}\right)$ is the number of these blue-green dual lines that are crossed ${ }^{4}$ by the segment $\tilde{\rho}_{1}^{*} \tilde{\rho}_{2}^{*}$.

Put $r_{\mathrm{c}}=\left|R_{\mathrm{c}}\right|$, and choose a parameter $\xi=a \sqrt{r_{\mathrm{c}}}$, for an appropriate absolute constant a. Construct a $(1 / \xi)$-cutting of $K$, which consists of $O\left(\xi^{2}\right)=O\left(r_{\mathrm{c}}\right)$ cells (see [13] and [18] for details concerning cuttings). Each cell is crossed by at most $|K| / \xi$ lines of $K$. Choose the constant $a$ so that the number of cells is smaller than $r_{\mathrm{c}}$. Then there exists a cell that contains at least two points $\widetilde{\rho_{1}^{*}}, \widetilde{\rho_{2}^{*}}$ of $\widetilde{R_{\mathrm{c}}^{*}}$, and, clearly, only lines of $K$ that cross that cell can cross the segment $\tilde{\rho}_{1}^{*} \tilde{\rho}_{2}^{*}$. Hence

$$
d\left(\rho_{1}, \rho_{2}\right) \leq \frac{|K|}{\xi}=O\left(\frac{b g}{\sqrt{r_{\mathrm{c}}}}\right)
$$

We conclude that

$$
\frac{t^{2}}{36}=O\left(\frac{b g}{\sqrt{r_{\mathrm{c}}}}\right)
$$

thus

$$
r_{\mathrm{c}}=O\left(\frac{b^{2} g^{2}}{t^{4}}\right) .
$$

In other words, we have shown that the number of clusters is at most $O\left(b^{2} g^{2} / t^{4}\right)$, and since each cluster contains at most $3 b / t$ lines, we obtain that

$$
\left|R^{+}\right|=O\left(\frac{b^{3} g^{2}}{t^{5}}\right)
$$

Any line in $R^{+}$can participate in at most $b$ cycles of $C$, since each such cycle must "use" a different blue line. Hence the overall number of cycles in $C$, taking into account also the lines in $R \backslash R^{+}$, is

$$
O\left(r t+\frac{b^{4} g^{2}}{t^{5}}\right)
$$

Choose $t=b^{2 / 3} g^{1 / 3} / r^{1 / 6}$. This parameter is in the range $[1, b]$ when

$$
\frac{g^{2}}{b^{2}} \leq r \leq b^{4} g^{2}
$$

If $r>b^{4} g^{2}$, we choose $t=1$ and obtain the bound $|C|=O\left(r+b^{4} g^{2}\right)=O(r)$. If $r<g^{2} / b^{2}$, we choose $t=b$ and obtain the trivial bound $|C|=O(r b)$ (as just noted,

\footnotetext{
${ }^{4}$ Thus $d(\cdot, \cdot)$ is the "crossing distance" in $\mathcal{A}(K)$, as studied, e.g., in [7]. In particular, it satisfies the triangle inequality, as promised earlier.
} 
any line of $R$ can participate in at most $b$ cycles of $C)$, which is also $O\left(r^{1 / 2} g\right)$. Hence, we obtain

$$
|C|=O\left(r^{5 / 6} b^{2 / 3} g^{1 / 3}+r^{1 / 2} g+r\right)
$$

where we remind the reader that we have assumed that $b \leq g$. This completes the proof of Lemma 4.1.

\section{A General Bound on the Number of Empty Cycles}

\subsection{Reducing to Trichromatic Cycles}

Let $\mathcal{L}$ be a set of $n$ lines in $\mathbb{R}^{3}$ in general position, and let $C$ denote the set of all empty triangular counterclockwise cycles in $\mathcal{L}$. Color each line of $\mathcal{L}$ red, blue, or green, independently, at random, with equal probabilities. Consider a cycle $c \in C$ of the form $\ell_{1} \prec \ell_{2} \prec \ell_{3} \prec \ell_{1}$. With probability $1 / 9$, each line is assigned a different color (we then refer to $c$ as trichromatic), so that in the cyclic order along $c$ we pass from a blue line to a red line to a green line and back to the blue line. The expected number of trichromatic cycles in $C$ of this type is $\frac{1}{9}|C|$. Hence, ignoring constant factors, it suffices to consider the case where $\mathcal{L}$ is the disjoint union $B \cup R \cup G$ of three subfamilies of roughly equal size (the expected size of each family is $n / 3$ ), and $C$ is a collection of counterclockwise trichromatic triangular cycles in $B \times R \times G$ whose $x y$-projections are faces of $\mathcal{A}\left(\mathcal{L}^{*}\right)$, so that, for each cycle in $C$, the blue line passes below the red line, which passes below the green line, which passes below the blue line. As above, we refer to these cycles as empty.

Before continuing, we note that a trivial upper bound on $|C|$, which has already been effectively used above, is

$$
|C| \leq 2 \min \{|B| \cdot|R|,|B| \cdot|G|,|R| \cdot|G|\} .
$$

This is shown by charging each $c \in C$ to, say, the blue-red vertex of $c^{*}$, and by noting that no blue-red vertex of $\mathcal{A}\left(\mathcal{L}^{*}\right)$ is charged more than twice. Repeating the argument for blue-green and red-green vertices yields (2).

Assign an orientation to each line of $\mathcal{L}$, so that each of the two possible orientations is chosen at random with probability $1 / 2$. Each cycle $c \in C$, formed by three lines $\ell_{1}, \ell_{2}, \ell_{3}$, has probability $1 / 8$ to be such that $c^{*}$ lies to the left of each of the projections $\ell_{1}^{*}, \ell_{2}^{*}, \ell_{3}^{*}$. Hence we may assume that all the cycles in $C$ have this property, which implies that the three orientations of the lines forming a cycle in $C$ cannot all lie in a common semicircle of the circle $\mathbb{S}^{1}$ of orientations.

Our goal is to decompose the problem of bounding the number of empty cycles into subproblems, each involving appropriate subsets of $B, R$, and $G$, so that these subsets satisfy conditions (C1) and (C2) of Lemma 4.1. Each cycle of $C$ will appear as an empty cycle in one of these subproblems, and the bound on $|C|$ will follow by summing up the bounds obtained by applying Lemma 4.1 to each subproblem separately. 


\subsection{Enforcing Conditions $(C 1)$ and $(C 2)$}

The decomposition of the problem into subproblems that satisfy conditions (C1) and (C2) of Lemma 4.1 is accomplished using fairly standard, albeit technically involved, space decomposition methods. We represent lines in 3-space as either points or hyperplanes in real projective 5-space, using Plücker coordinates [6], [20].

For technical reasons, we first decompose the problem into $O(1)$ subproblems, where in each subproblem the horizontal orientations of all the lines of $B$ (i.e., the orientations of the projected lines in $B^{*}$ within the $x y$-plane) lie in a fixed quadrant of $\mathbb{S}^{1}$, and similarly for $R$ and $G$. (These three quadrants need not be distinct.) Fix one of these subproblems, and continue to denote the subsets of $B, R$, and $G$ that belong to the subproblem by the same symbols. Recall that we need to ensure that the horizontal orientations have to come in counterclockwise order $B,-G, R,-B, G,-R$ (refer to Fig. 6), where we use $B, R, G$ to denote the intervals of orientations of the lines in $B^{*}, R^{*}, G^{*}$, respectively, and $-B,-R,-G$ to denote the corresponding antipodal intervals. Note that this condition is equivalent to the condition that the orientations of all lines in $R^{*}$ lie counterclockwise to those of all lines in $B^{*}$, which in turn lie counterclockwise to those of all lines in $G^{*}$, which in turn lie counterclockwise to those of all lines in $R^{*}$.

This implies that certain combinations of quadrants can be ignored, since this condition cannot arise at all for them. (For example, if $B$ is associated with the first quadrant, $R$ cannot be associated with the fourth quadrant, nor $G$ with the second quadrant. Similarly, we can ignore cases where all three quadrants lie in the same halfplane.) Moreover, if the quadrants associated with, say, $B$ and $R$ are adjacent, the desired separation of the horizontal orientations holds for every pair of lines in $B \times R$, and thus need not be enforced at all. For specificity, we consider the case where both $B$ and $R$ are associated with the first quadrant, and $G$ with the third quadrant. Here condition (C2) must be enforced for each pair of these subsets. The other cases are handled in a similar (and sometimes simpler) manner.

The decomposition in 5-space proceeds as follows. Let $b, r, g$ denote the respective sizes of $B, R, G$. Represent each line $\ell$ in 3-space, having the equations $y=a_{1} x+$ $a_{2}, z=a_{3} x+a_{4}$, by its Plücker point $q(\ell)$. Reordering the coordinates, reversing some signs, and passing to the real, rather than projective, space, we can put $q(\ell)=$ $\left(a_{1}, a_{2}, a_{3}, a_{4}, a_{1} a_{4}-a_{2} a_{3}\right)$; see [6] and [20]. In this parametrization we exclude lines parallel to the $y z$-plane, but we may assume that none of the lines in $\mathcal{L}$ are parallel to that plane. All the Plücker points lie on the Plücker surface $\Pi$, which in the coordinate system we have chosen is the quadric $x_{5}=x_{1} x_{4}-x_{2} x_{3}$.

The original decomposition of the horizontal orientations into fixed quadrants allows us to represent unambiguously the horizontal orientation of a line by its coefficient $a_{1}$. Specifically, in the subcase under consideration, for lines $\ell \in B, \ell^{\prime} \in R, \ell^{\prime \prime} \in G$, whose respective $a_{1}$-coefficients are $a, a^{\prime}, a^{\prime \prime}$, the clockwise/counterclockwise relations in condition (C2) can be expressed as $a^{\prime}>a^{\prime \prime}>a$.

We map each line $\ell^{\prime} \in R \cup G$ to a surface $\sigma\left(\ell^{\prime}\right)$ in $\mathbb{R}^{4}$, which is the union of two hyperplanes $\sigma_{1}$ and $\sigma_{2}$, where $\sigma_{1}$ is the locus of all (points representing) lines $\ell$ that meet $\ell^{\prime}$, and $\sigma_{2}$ is the locus of all points representing lines whose $x y$-projection is parallel to that of $\ell^{\prime}$ (i.e., they have the same $a_{1}$-coefficient as $\ell^{\prime}$ ). Each of these conditions does indeed correspond to a hyperplane in 4 -space. Note that $q(\ell)$ lies below (resp., above) 
$\sigma_{1}\left(\ell^{\prime}\right)$, relative to the fourth coordinate direction, if and only if $\ell$ passes below (resp., above) $\ell^{\prime}$ in 3-space. Similarly, if $\ell^{\prime}$ belongs to $R$ then $q(\ell)$ lies to the left (resp., right) of $\sigma_{2}\left(\ell^{\prime}\right)$, relative to the first coordinate direction, if and only if the horizontal orientation of $\ell$ lies clockwise (resp., counterclockwise) to $\ell^{\prime}$. If $\ell^{\prime}$ belongs to $G$ then the latter property holds with clockwise and counterclockwise interchanged.

Let $\Sigma_{R \cup G}$ denote the collection of the $2 r+2 g$ hyperplanes that constitute the surfaces $\sigma\left(\ell^{\prime}\right)$, for $\ell^{\prime} \in R \cup G$. Fix a parameter $\xi$, to be determined later, and construct a $(1 / \xi)$ cutting of $\mathcal{A}\left(\Sigma_{R \cup G}\right)$ in $\mathbb{R}^{5}$, which is a decomposition of 5-space into simplices, each crossed by at most $(2 r+2 g) / \xi$ hyperplanes, and extract from it all the cells that are crossed by the Plücker surface $\Pi$. Standard machinery [1] implies that there exists such a cutting for which the number of cells crossed by $\Pi$ is $O\left(\xi^{4} \log \xi\right)$. Because of the general position assumption, the cutting can be constructed so that no point representing a line in $B$ lies on the boundary of any full-dimensional simplex. Moreover, the cutting can be constructed in such a manner that each simplex is crossed by at most $2 r / \xi$ hyperplanes corresponding to lines in $R$, and by at most $2 g / \xi$ hyperplanes corresponding to lines in $G$. Also, by partitioning cells further as needed, we may assume that each cell contains at most $b /\left(\xi^{4} \log \xi\right)$ points corresponding to the lines in $B$. This further partitioning does not change the asymptotic bound on the number of cells of the cutting.

For each cell $\tau$ of the cutting, let $B_{\tau}$ denote the set of lines $\ell \in B$ whose corresponding points $q(\ell)$ lie in $\tau$; let $R_{\tau}$ (resp., $G_{\tau}$ ) denote the set of lines $\ell^{\prime} \in R$ (resp., $\ell^{\prime} \in G$ ) such that (at least one of the two hyperplanes of) $\sigma\left(\ell^{\prime}\right)$ crosses $\tau$. Finally, let $R_{\tau}^{0}$ denote the set of lines $\ell^{\prime} \in R$ such that $\tau$ lies fully below $\sigma_{1}\left(\ell^{\prime}\right)$ and fully to the left of $\sigma_{2}\left(\ell^{\prime}\right)$. Similarly, let $G_{\tau}^{0}$ denote the set of lines $\ell^{\prime} \in G$ such that $\tau$ lies fully above $\sigma_{1}\left(\ell^{\prime}\right)$ and fully to the right of $\sigma_{2}\left(\ell^{\prime}\right)$.

Fix a cell $\tau$ of the cutting. By the reduction described in the preceding subsections, and by construction, each cycle in $C$ that involves a line $\ell \in B_{\tau}$ must involve a red line in $R_{\tau} \cup R_{\tau}^{0}$ and a green line in $G_{\tau} \cup G_{\tau}^{0}$.

Consider first cycles in $\left(B_{\tau} \times R_{\tau} \times G_{\tau}\right) \cup\left(B_{\tau} \times R_{\tau} \times G_{\tau}^{0}\right) \cup\left(B_{\tau} \times R_{\tau}^{0} \times G_{\tau}\right)$. Using (2), the number of such cycles is at most

$$
O\left(\frac{b}{\xi^{4} \log \xi} \cdot\left(\frac{r}{\xi}+\frac{g}{\xi}\right)\right)=O\left(\frac{n^{2}}{\xi^{5} \log \xi}\right) .
$$

Multiplying by the total number of cells $\tau$, the overall number of cycles of this type is at most $O\left(n^{2} / \xi\right)$.

The main task is analyzing cycles in $B_{\tau} \times R_{\tau}^{0} \times G_{\tau}^{0}$. Consider the two sets $R_{\tau}^{0}$ and $G_{\tau}^{0}$. As is easily verified, the sets $B_{\tau}, R_{\tau}^{0}, G_{\tau}^{0}$ satisfy conditions (C1) and (C2) of Lemma 4.1, with the exception that the interactions between the lines in $R_{\tau}^{0}$ and in $G_{\tau}^{0}$ are not as yet determined. In more detail, (i) each line in $B_{\tau}$ passes below each line in $R_{\tau}^{0}$ and above each line in $G_{\tau}^{0}$, and (ii) the horizontal orientation of each line in $B_{\tau}$ lies clockwise to that of each line in $R_{\tau}^{0}$, and counterclockwise to that of each line in $G_{\tau}^{0}$. The above/below relationship between the lines of $R_{\tau}^{0}$ and those of $G_{\tau}^{0}$, as well as the clockwise/counterclockwise relation between their horizontal orientations are still not determined.

We next apply another cutting-based partitioning scheme to $R_{\tau}^{0}$ and $G_{\tau}^{0}$, whose purpose is to enforce the missing relationships between the red and green lines. Specifically, map the lines in $R_{\tau}^{0}$ into Plücker points in $\mathbb{R}^{5}$, and the lines in $G_{\tau}^{0}$ into pairs of hyperplanes, 
as above. Choose a parameter $\lambda$, to be determined later, construct a $(1 / \lambda)$-cutting of the arrangement of the green surfaces $\sigma(\ell)$, for $\ell \in G_{\tau}^{0}$, and extract its $O\left(\lambda^{4} \log \lambda\right)$ cells that are crossed by $\Pi$. As above, we may assume that each cell contains at most $\left|R_{\tau}^{0}\right| /\left(\lambda^{4} \log \lambda\right)$ red points of $R_{\tau}^{0}$, and is crossed by at most $\left|G_{\tau}^{0}\right| / \lambda$ green surfaces. For each cell $\varphi$ of the cutting, let $R_{\tau}^{0}(\varphi)$ be the set of lines $\ell \in R_{\tau}^{0}$ such that $q(\ell) \in \varphi$, let $G_{\tau}^{0}(\varphi)$ be the set of lines $\ell^{\prime} \in G_{\tau}^{0}$ such that $\sigma\left(\ell^{\prime}\right)$ crosses $\varphi$, and let $G_{\tau}^{-}(\varphi)$ denote the set of lines $\ell^{\prime} \in G_{\tau}^{0}$ such that $\varphi$ lies fully below $\sigma_{1}\left(\ell^{\prime}\right)$ and to the right of $\sigma_{2}\left(\ell^{\prime}\right)$.

Again, by the preceding reductions and by construction, for any cycle $c \in C \cap\left(B_{\tau} \times\right.$ $\left.R_{\tau}^{0} \times G_{\tau}^{0}\right)$ there exists a cell $\varphi$ such that $c$ is either in $C \cap\left(B_{\tau} \times R_{\tau}^{0}(\varphi) \times G_{\tau}^{0}(\varphi)\right)$ or in $C \cap\left(B_{\tau} \times R_{\tau}^{0}(\varphi) \times G_{\tau}^{-}(\varphi)\right)$. Using (2), the number of cycles in $C \cap\left(B_{\tau} \times R_{\tau}^{0}(\varphi) \times G_{\tau}^{0}(\varphi)\right)$ is at most

$$
\left|R_{\tau}^{0}(\varphi)\right| \cdot\left|G_{\tau}^{0}(\varphi)\right| \leq \frac{r}{\lambda^{4} \log \lambda} \cdot \frac{g}{\lambda}=O\left(\frac{n^{2}}{\lambda^{5} \log \lambda}\right) .
$$

Summing this bound over all cells $\varphi$, and over all cells $\tau$ in the original cutting, the number of cycles of this type is at most $O\left(\xi^{4} n^{2} \log \xi / \lambda\right)$.

Finally, we turn to the analysis of the number of cycles in $C \cap\left(B_{\tau} \times R_{\tau}^{0}(\varphi) \times G_{\tau}^{-}(\varphi)\right)$. Observe that $B_{\tau} \cup R_{\tau}^{0}(\varphi) \cup G_{\tau}^{-}(\varphi)$ satisfies the assumptions of Lemma 4.1. We thus have that the total number of cycles in $C \cap\left(B_{\tau} \times R_{\tau}^{0}(\varphi) \times G_{\tau}^{-}(\varphi)\right)$ is proportional to

$$
\begin{aligned}
\left|R_{\tau}^{0}(\varphi)\right|^{5 / 6}\left|B_{\tau}\right|^{1 / 3}\left|G_{\tau}^{-}(\varphi)\right|^{1 / 3} \min \left\{\left|B_{\tau}\right|,\left|G_{\tau}^{-}(\varphi)\right|\right\}^{1 / 3} \\
+\left|R_{\tau}^{0}(\varphi)\right|^{1 / 2} \max \left\{\left|B_{\tau}\right|,\left|G_{\tau}^{-}(\varphi)\right|\right\}+\left|R_{\tau}^{0}(\varphi)\right|,
\end{aligned}
$$

where

$$
\begin{aligned}
\left|B_{\tau}\right| & \leq \frac{b}{\xi^{4}} \leq \frac{n}{\xi^{4}}, \\
\left|R_{\tau}^{0}(\varphi)\right| & \leq \frac{r}{\lambda^{4}} \leq \frac{n}{\lambda^{4}}, \quad \text { and } \\
\left|G_{\tau}^{-}(\varphi)\right| & \leq g \leq n .
\end{aligned}
$$

Substituting these estimates into the bound above, we obtain

$$
\left|C \cap\left(B_{\tau} \times R_{\tau}^{0}(\varphi) \times G_{\tau}^{-}(\varphi)\right)\right|=O\left(\frac{n^{11 / 6}}{\xi^{8 / 3} \lambda^{10 / 3}}+\frac{n^{3 / 2}}{\lambda^{2}}+\frac{n}{\lambda^{4}}\right) .
$$

Multiplying this expression by the number of cells $\tau, \varphi$, and adding the estimates for the remaining types of cycles, we conclude that the overall number of cycles in $C$ is

$$
O\left(\frac{n^{2}}{\xi}+\frac{n^{2} \xi^{4} \log n}{\lambda}+\xi^{4 / 3} \lambda^{2 / 3} n^{11 / 6} \log ^{2} n+\xi^{4} \lambda^{2} n^{3 / 2} \log ^{2} n+\xi^{4} n \log ^{2} n\right) .
$$

Choose $\lambda=\xi^{5} \log n$ to obtain

$$
|C|=O\left(\frac{n^{2}}{\xi}+\xi^{14 / 3} n^{11 / 6} \log ^{8 / 3} n+\xi^{14} n^{3 / 2} \log ^{4} n+\xi^{4} n \log ^{2} n\right) .
$$

Now choose $\xi=n^{1 / 34} / \log ^{8 / 17} n$ to get $|C|=O\left(n^{2-1 / 34} \log ^{8 / 17} n\right)$. We have thus arrived at the main result of this section, which provides the missing ingredient for the proof of Theorem 3.1. 
Theorem 5.1. Given a set $\mathcal{L}$ of n nonvertical lines in $\mathbb{R}^{3}$ in general position, the number of empty triangular counterclockwise cycles defined by $\mathcal{L}$ is $O\left(n^{2-1 / 34} \log ^{8 / 17} n\right)$.

\section{Acknowledgments}

The authors express their gratitude to Pankaj K. Agarwal, Sariel Har-Peled, and Shakhar Smorodinsky for insightful suggestions concerning the material presented in this paper.

\section{References}

1. P. K. Agarwal and J. Matoušek, On range searching with semialgebraic sets, Discrete Comput. Geom. 11 (1994), 393-418.

2. M. de Berg, Ray Shooting, Depth Orders and Hidden Surface Removal, Lecture Notes in Computer Science, 703, Springer-Verlag, Berlin, 1993.

3. M. de Berg, M. van Kreveld, M. Overmars, and O. Schwarzkopf, Computational Geometry: Algorithms and Applications, Springer-Verlag, Berlin, 2000.

4. E. Catmull, A Subdivision Algorithm for Computer Display of Curved Surfaces, Ph.D. Thesis, UTECCSC-74-133, Department of Computer Science, University of Utah, 1974.

5. B. Chazelle, H. Edelsbrunner, L. J. Guibas, R. Pollack, R. Seidel, M. Sharir, and J. Snoeyink, Counting and cutting cycles of lines and rods in space, Comput. Geom. Theory Appl. 1 (1992), 305-323.

6. B. Chazelle, H. Edelsbrunner, L. Guibas, M. Sharir, and J. Stolfi, Lines in space: combinatorics and algorithms, Algorithmica 15 (1996), 428-447.

7. B. Chazelle and E. Welzl, Quasi-optimal range searching in spaces of finite VC-dimension, Discrete Comput. Geom. 4 (1989), 467-489.

8. K. Clarkson and P. Shor, Applications of random sampling in computational geometry, II, Discrete Comput. Geom. 4 (1989), 387-421.

9. S. E. Dorward, A survey of object-space hidden surface removal, Internat. J. Comput. Geom. Appl. 4 (1994), 325-362.

10. H. Fuchs, Z. M. Kedem, and B. Naylor, On visible surface generation by a priori tree structures, Comput. Graph. 14 (1980), 124-133.

11. A. Gaifullin, Two isotopic weavings one of which is realizable and the other is not, Manuscript, 2002

12. S. Har-Peled and M. Sharir, On-line point location in planar arrangements and its applications, Discrete Comput. Geom. 26 (2001), 19-40.

13. J. Matoušek, Lectures on Discrete Geometry, Springer Verlag, New York, 2002.

14. M. H. Overmars and M. Sharir, A simple output-sensitive algorithm for hidden surface removal, $A C M$ Trans. Graphics 11 (1992), 1-11.

15. J. Pach, R. Pollack and E. Welzl, Weaving patterns of lines and line segments in space, Algorithmica 9 (1993), 561-571.

16. M. S. Paterson and F. F. Yao, Efficient binary space partitions for hidden-surface removal and solid modeling, Discrete Comput. Geom. 5 (1990), 485-503.

17. D. Repovš, A. Skopenkov, and F. Spaggiari, An infinite sequence of non-realizable weavings, Preprint series, Department of Mathematics, University of Ljubljana, 2002.

18. M. Sharir and P. K. Agarwal, Davenport-Schinzel Sequences and Their Geometric Applications, Cambridge University Press, New York, 1995.

19. A. Solan, Cutting cycles of rods in space, Proc. 14th Annu. ACM Sympos. Comput. Geom., 1998, pp. 135142.

20. D. M. Y. Sommerville, Analytic Geometry of Three Dimensions, Cambridge University Press, Cambridge, 1934.

21. I. E. Sutherland, R. F. Sproull, and R. A. Schumacker, A characterization of ten hidden-surface algorithms, ACM Comput. Surv. 6 (1974), 1-55.

Received April 2, 2003, and in revised form March 29, 2004. Online publication October 29, 2004. 